\title{
An Analytical Model for Dynamic Spectrum Sensing in Cognitive Radio Networks using Blockchain Management
}

Nikhil Marriwala ( $\nabla$ nikhilmarriwala@gmail.com )

U.I.E.T https://orcid.org/0000-0002-1093-630X

Simran Taneja

Kurukshetra University University Institute of Engineering and Technology

\section{Research Article}

Keywords: Blockchain, CRN Security, Error Rate, NES Algorithm

Posted Date: October 21st, 2021

DOl: https://doi.org/10.21203/rs.3.rs-949124/v1

License: (c) (i) This work is licensed under a Creative Commons Attribution 4.0 International License.

Read Full License 


\section{Abstract}

The recent advances in wireless communication have led to the problem of growing spectrum scarcity. The problem of spectrum allocation is due to advance research in wireless communication. As new wireless applications are emerging, day after another, and making use of the available wireless spectrum for communication, the demand for spectrum increase makes the available spectrum scarcer. Mostly part of the spectrum is not utilized significantly in the wireless network. Cognitive Radio (CR) is a new technology that enables an unlicensed secondary user to coexist with licensed primary users in licensed spectrum bands without inducing interference to licensed primary users communication. This technology can significantly ease the spectrum redundancy problem \& enhance the efficiency of utilization of spectrum. Cognitive Radio Networks (CRN) or Dynamic Spectrum Access Networks are formed by several CR nodes and they are often called NeXt Generation (XG) communication networks. This XG communication network is expected to give high transfer speed to versatile clients through heterogeneous remote designs and dynamic range access procedures. CRNs have drawn in incredible exploration interest in the new years. Nonetheless, research on the security parts of CRNs has been exceptionally restricted. As CRN is like a remote organization, the idea of the remote media is outside, it is more helpless against assaults when contrasted with that of a wired organization. This channel might be stuck/abuse due to remote media information is to be listened to.

\section{Introduction}

Blockchain adoption is gaining huge momentum as the industry is evolving at a fast pace. Many startups have initiated use cases and there is a significant rise in investments in blockchain projects as well. Although blockchain is still evolving in terms of technological maturity, innovative experimental adoption and customization are continuously rising. Blockchain has the potential of displacing a setup innovation and stirs up the business or a pivotal item that makes a totally new industry and initial trends with the rise of cryptocurrencies has signalled that blockchain has been disruptive for the banking industry and financial services.

Cryptocurrency has the potential of shaking a centralized banking system by eliminating the need to pay fees for using credit or debit cards. Recent Trends have shown that blockchain is turning out to be a sustaining technology rather disruptive and has huge potential in the supply chain, healthcare, Internet of Things (IoT), education and public services. Commercial viability and acceptability of blockchain are on the rise and 3021 patent families related to blockchain applications are divided into four sub-categories based on different types of applications like Payments and Transaction systems, Financial services business, Administration and E-commerce. Extensive use of cryptography and decentralization makes it highly secure. Privacy issues over public blockchains can be addressed by implementing blockchain in a controlled manner (permissioned blockchain). Blockchains can be named public, private or half and half variations, contingent upon their application [4].

- Public - No one owns Public blockchains, they are fully decentralized and are visible by anyone. 
- Private - These are also referred to as permissioned blockchains and uses privilege to control who can peruse from and write to the blockchain.

- Hybrid - These blockchains are public just to an advantaged bunch. Agreement measure is constrained by special workers utilizing a bunch of rules consented to by all gatherings.

Blockchain may record exchanges, contracts, data resources or for all intents and purposes whatever can be put away in advanced structure. Blockchain records are lasting, carefully designed, straightforward, and accessible. Each new "block" created is added to the furthest limit of a "chain." Initiation, validation, storage, and distribution of each new block is managed by a protocol. Blockchain replaces the need for third-party intermediaries and participants of blockchain run complex algorithms to certify the integrity of records in the block.

Blockchain hence is an interesting alternative to how data is stored. Databases have been the way to store data in databases. Though databases are quite fast and user friendly, they too have limitations and issues like lack of immutability. The following table highlights key differences between blockchain and databases [5].

Table 1

Comparison Of Databases With Blockchain [5]

\begin{tabular}{|c|c|c|}
\hline Criteria & Databases & Blockchain \\
\hline \multirow[t]{2}{*}{ Data Integrity } & Records and Data items can be changed. & $\begin{array}{l}\text { Transaction data is immutable, } \\
\text { can't be }\end{array}$ \\
\hline & & changed after the transaction. \\
\hline Authority & $\begin{array}{l}\text { Administrator or selected people have } \\
\text { authoritative control }\end{array}$ & No central authority. \\
\hline Transparency & $\begin{array}{l}\text { All transactional data is hidden from each } \\
\text { other. }\end{array}$ & $\begin{array}{l}\text { All transactional data is open for } \\
\text { everyone. }\end{array}$ \\
\hline Cost & Implementation Process is costly. & $\begin{array}{l}\text { It can help in cutting down } \\
\text { excessive costing. }\end{array}$ \\
\hline Performance & They are comparatively faster. & They are slow. \\
\hline \multirow[t]{2}{*}{ Trust } & $\begin{array}{l}\text { Suitable for organizations having mutual } \\
\text { trust. }\end{array}$ & $\begin{array}{l}\text { Suitable for organizations where } \\
\text { people don't }\end{array}$ \\
\hline & & trust each other. \\
\hline
\end{tabular}

Blockchain is a system having various components that work together to perform transactions of business operations. Each element in the blockchain has a specific role to play as listed below [4].

- Node: Node is a computing device within a blockchain that can initiate, receive or validate a transaction. A node operates with the help of a software application that offers various 
functionalities related to the business use case. Blockchain typically has two kinds of nodes i.e., validator nodes and member nodes. Validator nodes have more capabilities as compared to member nodes as they could initiate, received \& validated a transaction whereas defined nodes can only initiate \& receive a transaction.

- Transaction: Transaction in a blockchain is a collection of various data items that carry facts about the exchange of something like a product, service, entity, event or anything that carries value. For example, it could be the transfer of ownership, issue of certificate etc.

- Block: A Block is a data structure that keeps a set of transactions. Every block on successful verification is distributed to all nodes of the blockchain network. A block contains various elements as shown in Table 2. Block number can be used for the unique identification of blocks in a chain of blocks. A nonce is a random number that helps generate a hash code with reasonably difficult mathematical computation. Previous represents the hash code of the previous block and Hash represents the hash code of the current block.

- Chain: Chain in a Blockchain is an interconnected sequence of blocks in chronological order with the latest block in the last with each block having reference to its previous block to trace back the complete blockchain.

Table 2

Block Structure in A Blockchain Radio Networks Summary

[5]

A.

\begin{tabular}{|llllll|}
\hline Block & \# 1 & & & & \\
\hline Nonce & 36584 & & & & \\
\hline Transaction & Rs 65 & from & Ravi & to & Mohit \\
\cline { 2 - 6 } & Rs 30 & from & Pankaj & To & Vishal \\
\cline { 2 - 6 } & Rs 45 & from & Ravi & To & Mohit \\
\cline { 2 - 6 } & Rs 50 & from & Rohit & To & Sonu \\
\cline { 2 - 6 } & Rs 30 & from & Mohit & To & Kunal \\
\hline Previous & 00000000000000000000000 & \\
\hline Hash & \multicolumn{4}{l}{$0000 \mathrm{ab2des} 234 \mathrm{f} 453 \mathrm{f} 4 \mathrm{r} 5 \mathrm{tfe} 34$} & \\
\hline
\end{tabular}

B. 


\begin{tabular}{|llllll|}
\hline Block & \# 2 & & & & \\
\hline Nonce & 82549 & & & & \\
\hline Transaction & Rs 25 & from & Mohit & to & Priya \\
\cline { 2 - 6 } & Rs 30 & from & Sonu & to & Reena \\
& Rs 15 & from & Kunal & to & Ravi \\
& Rs 25 & from & Ravi & to & Sonu \\
& Rs 30 & from & Pankaj & to & Kunal \\
\hline Previous & 0000ab2des234f453f4r5tfe34 & \\
\hline Hash & \multicolumn{4}{l}{ 0000rgtf3r4r45t6y6hfrett56y5 } & \\
\hline
\end{tabular}

Miners: a blockchain may have two kinds of nodes Miner nodes (also called Network nodes) and User Nodes. Miners are the one who has a copy of the blockchain and they also participate in the block verification process, whereas User nodes can only initiate a transaction [5].

Consensus: Consensus algorithms are used to assure necessary conformity on a single state of the network consisting of distributed machines or network nodes in a blockchain system. The consensus is assessed by comparing the hash codes of all copies of the chain. This assessment involves mathematical problem solving that requires a lot of computing power. For this provision of reward is required for people to contribute resources for maintaining the network. Following are some popular consensus algorithms

- Proof of work (PoW) mechanism: In this mechanism, all the network nodes or participants on the network fetch recent transactions as a block. Each block within the chain must have the hash code of the previous block. To select a network node who will be assigned to process the block, a reasonably complex mathematical problem is created. The node that solves the given mathematical problem first is allowed to process the block. For doing this job, the network node is rewarded which could be a transaction fee or some bitcoin. The complexity of the mathematical problem increases after every successful transaction i.e. the network node needs to compute larger hash codes. The complexity of the mathematical problem is also determined by the number of network nodes in the blockchain network [4].

- Proof of stake is a mechanism that requires network nodes to invest a native currency to perform a transaction i.e. users need to possess a stake in the transaction. For example, ether is the native currency in the Ethereum blockchain, and a fraction of ether is invested by network nodes for the processing of transactions and achieving consensus.

- Proof of activity this scheme of consensus combination of work proof \& stake proof mechanism s. A random number of network nodes have to perform complex mathematical problems and need to digitally sign the block using a crypto key to make a block official. 
- Proof of capacity This scheme is dependent on the idea of the contribution of storage and computation resources by all network nodes. This requires network nodes to allocate a portion of their hard drive for block verification, while proof of storage requires network nodes to reserve a portion of disk space either in the machine or a distributed cloud.

- Ripple (XRP) Ripple follows a different approach and involves social networks for achieving consensus on the basis of the value of network nodes. This value is computed on the basis of the number of unique nodes one connects to. This may encourage biasing where newcomers need social intelligence and reputation to participate. This scheme is more suitable for permissioned or private blockchain systems [5].

\section{li. Blockchain Use Case Selection And Design Process}

Blockchain use case selection involves many decisions based on the following criteria and NITI Ayog has published a blockchain use case selection framework [4] as shown in Figure 2 that covers all these.

- Is there a compelling business case to reduce intermediaries?

- Are Multiple Stakeholders Involved?

- Are we working with advanced resources rather than actual Assets?

- Do numerous gatherings require shared compose access?

- Do we require superior and fast exchanges?

- Do we plan to store non-conditional information as a feature of your answer?

- Do we need to depend on believed parties for consistent reasons?

- Do we have a powerful Tokenomics Model?

- Do we need the capacity to control usefulness?

- Should exchanges be public?

- The structure presents the following four potential results dependent on models,

- Don't use blockchain

Blockchain can't do this effectively yet solutions are in development shown Fig. 3

- A strong case for public blockchain

- A strong case for private blockchain

From that point onward, an assortment of plan choices around blockchain arrangement should be made, similar to the sort of blockchain, agreement convention, block size and recurrence. The bolts just represent one of the potential groupings to settle on plan choices. A few choices are required to set up acceptable levels of expansion (like block size and rate of occurrence), security, cost of processing and performance [5]. 


\section{lii. Blockchain Based Crn Security Technique}

Block chaining is a basic innovation preoccupied via Bit-coined. It is another use of conventional innovation in the web period, that incorporates conveyed information stockpiling innovation, remote organizations, agreement system and cryptography [29]. As a decentralization publically data set, blockchaining utilizes publically keying cryptographical calculations, hashing capacities, agreement instruments \& different innovations to fabricate a decentralization non-verification framework that could be utilized in internet business to guarantee client data security. Block chaining could be broadly utilized in web nance or a more extensive market. It would additionally advance the course of monetary globalisation \& would enormously affect the current monetary marketing design \& surprisingly the social design [30].

Block chained innovation enjoys the benefits of least exchange costing, solid straightforwardness, \& highest securities. It could adequately work on the proficiency of data usage, make out the exchange interaction straightforward, share management, and secure the authentic rights and interests, all things considered, to the exchange. Normal issues like a significant expense, low effectiveness, and low information stockpiling security in the normalized data set give groundbreaking thoughts [31]. Blockchain is a sort of carefully designed, full history information base stockpiling innovation, ordinarily utilizes highlight direct innovation toward coordinate every hub. Every hub understands the elements of steering, new hub distinguishing proof and information dispersal via multicast. It could show up at any hub in the framework. By utilizing cryptography's, it can create relating information blockings. The created information could look at the legitimacy of the data, \& could likewise understand the solid connection via the following information [32]. In remote organization correspondence, blockchain innovation is a sort of innovation that can't be altered among similar level auxiliary clients who don't confide in one another or have feeble trust without go-between investment. With respect to authentic cooperation in the information base as a publically record books, every hub storing the chronicled association recording of the entire organization, \& records of information assortment, exchange, flow and computation and examination are kept on the blockchain, which causes the nature of the information to acquire exceptional solid trust support, and guarantees the rightness of information investigation outputs \& the impact of mined data [33]. Taking into account the benefits of blockchain innovation, this chapter gives a psychological remote organization security calculation dependent on block-chaining

\section{A. Algo Design Structure}

The examination objects of such articles is a 2-waying confirmation issue bet ${ }^{n}$ the block chained loT gadget $\&$ the psychological remote organization combination focus.

Thusly, the framework in this article is basically made out of the blockchain framework, the psychological remote organization combination focus, \& the block chaining loT gadget. Clients speak via the block chaining framework via the combination place. 
The particular construction is displayed in Fig. 3 The l.o.T gadget sending hub data to the combination place, \& the combination community inquiries the blockchain framework for the presence of its hub data, \& afterwards the hub sending the information endorsed via the privately keying to the combination place, $\&$ checks whenever the detecting hub had a relating privately keying paired signature it. Assuming it is, forward the hub's solicitation to the block chaining framework, and forward the reaction of the block chained framework back to the detecting hub. The information endorsed by the detecting hub can affirm the character of partaking in range detecting, and can likewise guarantee that its information has not been altered or produced.

\section{Iv. Blockchain Technology Blockchain Based Secure Spectrum}

This report presents the blockchain innovation and notoriety system into the range detecting measure. Another safe range detecting strategy is proposed. This security detecting strategy incorporates the assessment of the client's immediate standing and suggestion notoriety. At the point when a helpful hub solicitation to get to a specific recurrence band, it needs to detect whether the recurrence band is inactive. In case it is inactive, it will send a proposal solicitation to the combination place. To stay away from conspiracy assault and malevolent hub conduct, the detecting results are more precise. Utilizing the blockchain innovation, the verifiable detecting records in the data set and the distance of cooperation history is viewed as a public record, which can be shared by each neighbour hub, and no hub in this situation can change the record data. The particular working course of safety range detecting dependent on blockchain innovation is displayed in Figure 4. After the combination place sends a discernment demand, the assessment of a hub's nearby standing worth is identified with the friend requester. It is important to acquire the requester's discernment computation record from the public record book of the blockchain to ascertain its immediate standing worth. For the sender, the neighbourhood direct standing worth is determined by the recipe

$$
T_{i}=\frac{N_{i}}{k} \theta \omega_{i}
$$

\subsection{4}

In equation 3.14, $T_{-}$i addresses the ith detecting period, $\mathrm{N}_{-} \mathrm{i}$ is the quantity of all the detecting periods up to $T_{-} i$ time-frame. The significance of $k$ is equivalent to and showing the right number of intuitive detecting in history [34], $\theta$ implies the detecting activity force, and its worth is determined by the recipe (14), $\omega_{-}$i is the impact coefficient of the detecting times in the public record book, and its worth is determined by equation 3.15 .

$$
\theta=1-e\left|-\frac{k}{m n}\right|
$$




$$
\omega_{i}=\sum_{i=1}^{n}\left|\frac{h_{1}}{m} \cdot \frac{l}{n}\right|
$$

\subsection{6}

where $h \_1$ is the quantity of cooperations in I period and $m$ is the size of the detecting period in every period, $\mathrm{n}$ is the all-out association time frame. It tends to be seen from the equation that the nearer is the cooperation history in the public record book, the extent will be the more prominent, and the effect on trust worth will increment as needs be [35].

At the point when the trust worth of the hub isn't in the front line, everything being equal, the hub is chosen by figuring the extensive trust esteem, and the thorough trust worth of the hub is determined by (3.17).

$$
T_{i, c}=\varnothing T_{i}+\phi\left|T_{i}+\theta \sum_{i} w_{i}\right|
$$

\subsection{7}

The security range detecting innovation dependent on the blockchain, through the computation of the extensive trust worth of the hubs in the organization. An ultimate conclusion making measure utilizes the exemplary trust esteem dynamic calculation. The hubs in the organization are grouped by setting the relating dismissal limit and trust edge. On the off chance that the far-reaching trust esteem is not exactly the dismissal limit, the hub is viewed as a malignant client; if the exhaustive trust esteem is more noteworthy than the trust edge, the hub is viewed as a typical client; if the thorough trust esteem is between the dismissal edge and the trust edge, the hub is viewed as a hub to be noticed. The hub gets the option to take part in helpful detecting through the ceaseless update of the trust esteem.

\section{Result Analysis}

In this section, we have to discuss the result analysis of Blockchain technology in the cognitive radio network. The simulation parameter is discussed in Table 1 
Table 1

Simulation Parameter

\begin{tabular}{|lll|}
\hline $\begin{array}{l}\text { Sr. } \\
\text { No. }\end{array}$ & Parameter & Value \\
\hline 1 & Simulation Area Setting & A Circular area with a radius of $\mathrm{m}$ \\
\hline 2 & Primary User & $\begin{array}{l}\text { Place a primary user anywhere on the edge of the circular } \\
\text { area }\end{array}$ \\
\hline 3 & $\begin{array}{l}\text { Working Parameters of a primary } \\
\text { user }\end{array}$ & $\begin{array}{l}\text { BPSK signal with a power of } 100 \mathrm{MW} \text { and bandwidth of } \\
100 \mathrm{kHz} .\end{array}$ \\
\hline 4 & Number of Nodes & $\begin{array}{l}\text { Randomly place } 15 \text { nodes (5 nodes with SNR }=-18 \mathrm{~dB} \\
\text { and }-14 \mathrm{~dB} \text { respectively) }\end{array}$ \\
\hline 7 & Noise Settings & AWGN \\
\hline 8 & $\begin{array}{l}\text { Average Detection Times } \\
\text { Auxiliary Node }\end{array}$ & $\begin{array}{l}\text { Spectrum Detection Method of } \\
\text { Node Front End }\end{array}$ \\
\hline
\end{tabular}

The circular area with radius $m$ is defined as the simulation area of the network. The primary users are placed anywhere on the edge of the circular area. The signal power is $100 \mathrm{MW}$ and bandwidth of 100 $\mathrm{kHz}$. Similarly, 15 nodes are placed in the system. Beyond this SNR of these nodes are $-18 \mathrm{~dB}$ and $-14 \mathrm{~dB}$ respectively. The noise used in the proposed system is AWGN. The average detection time and auxiliary nodes are 10000 and 3 respectively. The energy detection method is used as spectrum detection.

The cognitive radio topology is defined in Fig. 5 . The different no. of primary and secondary users is placed in particular geometry. The range of the network lies between -500 to $500 \mathrm{~m}$. There is one primary user and multiple secondary users. The primary user is located at the centre of the area while secondary users are locating around the primary users with the specific pattern.

The Total error rate is shown in Fig. 6 . The total error rate starts at $15 \%$ for $1 \mathrm{sec}$. As the graph shows the total error rate increases to $20.05 \%$ with a time period of $2 \mathrm{sec}$. Suddenly, the total error rate is decreased to $10 \%$. The steady-state error is $10 \%$.

A similar Analysis of Security in Cognitive Radio is displayed in Fig. 7. It tends to be seen from Figure that when the NES and SSSB calculations are utilized in the mix, as expanding the number of associations between the detecting hub, combination focus and blockchain the board community, the security file of the psychological remote organization rises fundamentally quicker. At the point when the quantity of associations surpasses multiple times, the security list utilizing the SSSB calculation rose quicker than the wellbeing file utilizing just the NES calculation, which completely mirrors the adequacy of the SSSB calculation. This is on the grounds that the SSSB calculation joins blockchain innovation. It can extraordinarily further develop the counter-assault capacity and security capacity of a psychological 
remote organization. The security execution of the proposed calculation is better when contrasted with the NES+SSB calculation.

Similar Analysis of Energy Consumption among Proposed and NES Algorithm is shown Fig. 8. It tends to be seen from Figure that after the quantity of detecting cycles surpasses 60 , the energy utilization of the calculation in this paper is essentially not exactly that of the other exemplary calculations. The more the quantity of detecting cycles, the more drawn out the detecting time, the more energy will be saved.

Extraordinarily assist the psychological remote organization with broadening the functioning life. This is on the grounds that the calculation in this paper can generally choose the hub with the best presentation to take part in agreeable detecting on schedule, so it can save more energy when the detecting execution is superior to the conventional strategy. The energy utilization of the proposed calculation is better compared to when contrasted with the NES calculation.

The organization traffic result is displayed in Table 2. The various upsides of Query each Second (QPS) examinations the traffic use. To demonstrate that the new calculation doesn't build network overhead, an organization traffic test was intentionally done. Organization traffic test plans to test the calculation and no character in this segment. To check the distinction of net-work traffic between remote passageways.

Table 2

Network Traffic Test Results

\begin{tabular}{|llll|}
\hline QPS & $\begin{array}{l}\text { Direct interactive traffic } \\
(\mathrm{MB} / \mathrm{S})\end{array}$ & $\begin{array}{l}\text { Encrypted authentication Traffic } \\
(\mathrm{MB} / \mathrm{S})\end{array}$ & $\begin{array}{l}\text { Traffic Utilization } \\
(\%)\end{array}$ \\
\hline 1 & 1 & 1 & 100 \\
\hline 2 & 2 & 2.001 & 99.95 \\
\hline 5 & 5 & 5.002 & 99.96 \\
\hline 10 & 10.001 & 10.005 & 99.96 \\
\hline 20 & 20.002 & 20.011 & 99.955 \\
\hline 40 & 20.004 & 20.02 & 99.95 \\
\hline
\end{tabular}

This convention builds the organization traffic pressure. Organization traffic test empowers. On the off chance that the organization traffic is tried with if top, the inquiry will be sent straightforwardly to the blockchain. Contrast the solicitation traffic and the solicitation traffic confirmed by this calculation, Query each second (QPS) and compute the organization traffic usage. To make the solicitation opportune. To get a reaction, and hence more precisely measure the effect on the organization. The number of administration hubs chose by the machine is 1

\section{Conclusion}

In the pragmatic application situations of intellectual remote organizations, there are typically genuine mistakes when the hubs detecting the information, which causes the detecting esteems to go amiss from 
the ordinary reach, or a few hubs purposely, send some unacceptable information to the combination community. Accordingly, focusing on the security issue of noxious hub assault in an intellectual remote organization, this paper proposes the hub assessment and planning (NES) calculation and the Secure Spectrum Sensing, which respects the client's collaboration history and association distance as a public record book, and is overseen by the blockchain the executive's community, which is helpful for the combination place to call hubs with astounding execution to take an interest in agreeable detecting.

\section{Declarations}

*Funding

Funding information is not applicable as No funding was received.No funds, grants, or other support was received.

*Conflicts of interest/Competing interests (include appropriate disclosures)

There is no Conflicts of interest/Competing interests in this paper. All authors certify that they have no affiliations with or involvement in any organization or entity with any financial interest or non-financial interest in the subject matter or materials discussed in this manuscript.

*Availability of data and material (data transparency)

Data sharing is not applicable to this article as no datasets were generated or analysed during the current study.

\section{References}

1. Abbas, Sana-e-Zainab, S \& Wajahat 2010, 'An Efficient Algorithm for Secure \& Fair Dynamic Spectrum Access in Cognitive Radio Networks', Canadian Journal on Multimedia and Wireless Networks, vol. 1, no. 3, pp. 173-177.

2. Amarnathprabhakaran, A \& Manikandan, A 2013, 'An Efficient Communication and Security for Cognitive Radio Networks', International Journal of Advanced Research in Electrical, Electronics and Instrumentation Engineering, vol. 2, issue 4, pp. 1689-1696.

3. Anand Z Jin \& Subbalakshmi, KP 2008, 'An analytical model for primary user emulation attacks in cognitive radio networks', DySPAN 2008, 3rd IEEE Symposium, IEEE, pp. 1-6.

4. Atta \& Alireza 2012, 'A Survey of Security Challenges in Cognitive Radio Networks: Solutions and Future Research Direction', Proceeding of IEEE, pp. 3172-3186.

5. Bhattacharjee, Suchismita \& Ningrinla Marchang 2015, 'AttackResistant Trust-Based Weighted Majority Game Rule for Spectrum Sensing in Cognitive Radio Networks', International Conference on Information Systems Security, Springer, pp. 441-460. 
6. Bhattacharya, PP, Khandelwal, R, Gera, R \& Anjali Agarwal 2011, 'Smart radio Spectrum management for Cognitive radio', International journal of Distributed and parallel systems, vol. 2, no. 4, pp. 12-24.

7. Cabric Danijela M Mishra \& Brodersen, RW 2004, 'Implementation issues in specrtum sensing for cognitive radios. Signal Systems and Computers', Conference record of 38th Asilomer Conference, IEEE, vol. 1, pp. 772-776.

8. Chen, R, Park, J \& Reed, JH 2008, 'Toward secure distributed spectrum sensing in cognitive radio networks', Communications Magazine, IEEE, vol. 46, no. 4, , pp. 50-55.

9. Dubey Rajni, Sanjeev Sharma \& Lokesh Chouhan 2012, 'Secure and trusted algorithm for cognitive radio network', Ninth International Conference on Wireless and Optical Communications Networks (WOCN), IEEE, pp. 1-7.

10. Etkin, R, Parekh, A \& Tse, D 2005, 'Spectrum sharing for unlicensed bands', Proc. IEEEDySPAN 2005, IEEE, pp. 251-258.

11. FCC 2003, 'Notice for Proposed Rulemaking (NPRM 03-322)', Facilitating Opportunities for flexible, Efficient, and Reliable Spectrum Use Employing Cognitive Radio Technologies. ET Docket, pp. 03108.

12. Feng Lin, Robert C Qiu, Zhen Hu, Shujie Hou, Lily Liy, James P Browningz \& Michael C Wicks 2012, 'Cognitive Radio Network as Sensors: Low Signal-to-Noise Ratio Collaborative Spectrum Sensing', Proceedings of Aerospace and Electronics Conference (NAECON), IEEE, pp. 978-985.

13. Harish Ganapathy, Constantine Caramanis \& Lei Ying 2010, 'Limited Feedback for Cognitive Radio Networks Using Compressed Sensing', IEEE 48th Annual Allerton Conference on Communication, Control, and Computing (Allerton), IEEE, p. 10901097.

14. Haykin \& Simon 2005, 'Cognitive radio: brain-empowered wireless Communication. Selected Areas in Communications', IEEE Journalon, vol. 23, no. 2, pp. 201-220.

15. Haykin \& Simon 2010, 'Cognitive radio: brain-empowered wireless communications', IEEE Journal of Selected Areas of Communication, vol. 2, pp. 201-220.

16. Ian F Akyildiz, Won-Yeol Lee \& Kaushik R Chowdhury 2006, 'Next generation/dynamic spectrum access/cognitive radio wireless networks: a survey', Computer networks, vol. 50, no. 13, pp. 21272159.

17. Januszkiewicz \& Lukasz 2010, 'Simplified human body models for interference analysis in the cognitive radio for medical body area networks', 8 th International conference on Medical Information and Communication Technology, IEEE, pp. 15-24.

18. Juebo \& Long Tang 2012, 'Research and Analysis on Cognitive Radio Network Security', Wireless Sensor Network, vol. 4, pp. 120-126.

19. Khuong Ho-Van \& Thiem Do-Dac 2018, 'Reliability-Security Tradeoff analysis of Cognitive Radio Networks with jamming and licensed interfernce', Wireless Communication and Mobile Computing, Hinadwi, vol. 2018, pp. 1-15.

20. Kwang Cheng Chen, Peng-Yu Chen, Neeli Prasad, Ying-Chang Liang \& Sumei Sun 2009, 'Trusted cognitive radio networking. Wireless Communications and Mobile Computing'. 
21. León, Olga, Juan Hernández-Serrano \& Miguel Soriano 2010, 'Securing cognitive radio networks', International journal of communication systems no. 5, pp. 633-652.

22. León, Olga, Juan Hernández-Serrano \& Miguel Soriano 2010, 'Securing cognitive radio networks', International Journal of Communication Systems, vol. 23, issue 5, pp. 633-652.

23. Mao, Huaqing \& Li Zhu 2011, 'An investigation on security of cognitive radio networks', International Conference on Management and Service Science (MASS), IEE, pp. 1-4.

24. Matteo Cesana, Francesca Cuomo \& Eylem Ekici 2010, 'Routing in cognitive radio networks: Challenges and solutions', Ad Hoc Networks, Elsevier., pp. 18-39.

25. McLoone, Safdar, GA \& O’Neillne, M 2009, 'Common Control Channel Security Framework for Cognitive Radio Networks', IEEE 69th, Vehicular Technology Conference, VTC Spring 2009, IEEE, pp. 26-29.

26. Meng, T 2015, 'Spatial Reusability-Aware Routing in Multi-Hop Wireless Networks', IEEE TMC, DOI 10.1109/TC.2015.2417543 .

27. Mitola, J \& Maguire, GQ 1999, 'Cognitive Radio: Making software radios more personal', IEEE personal Communications, vol. 6, no. 4 , pp. 13-18.

28. Muhammad Ayzed Mirza, Mudassar Ahmad, Muhammad Asif Habib,Nasir Mahmood, Nadeem Faisal, CM \& Usman Ahmad 2018, 'CDSS:Cluster-based distributed cooperative spectrum sensing model against primary user emulation cyber attack', The Journal of Supercomputing, Springer, Available Online, pp. 1-17.

29. Parvin Sazia \& Farookh Khadeer Hussain 2012, 'Trust-based security for community-based cognitive radio networks', IEEE 26th International Conference on Advanced Information Networking and Applications, IEEE, pp. 518-525.

30. Parvin, Sazia \& Farookh Khadeer Hussain 2011, 'Digital signature based secure communication in cognitive radio networks', Broadband and Wireless Computing, Communication and Applications (BWCCA), IEEE, pp. 230-235.

\section{Figures}




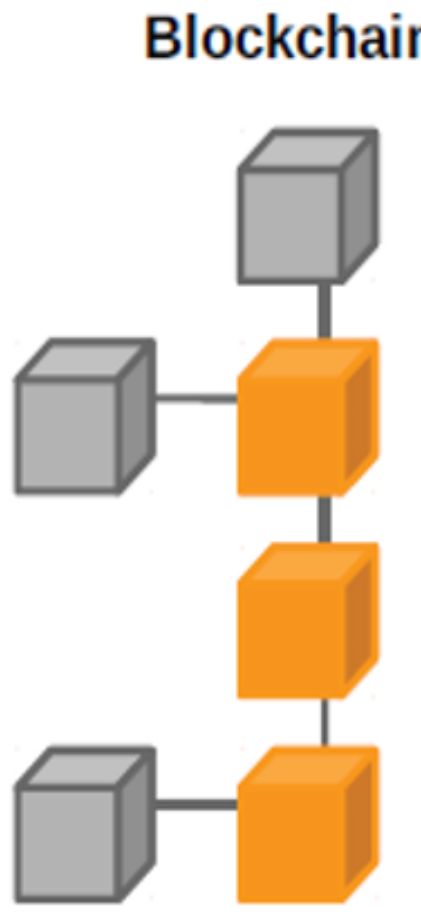

\section{Basic mechanisms}

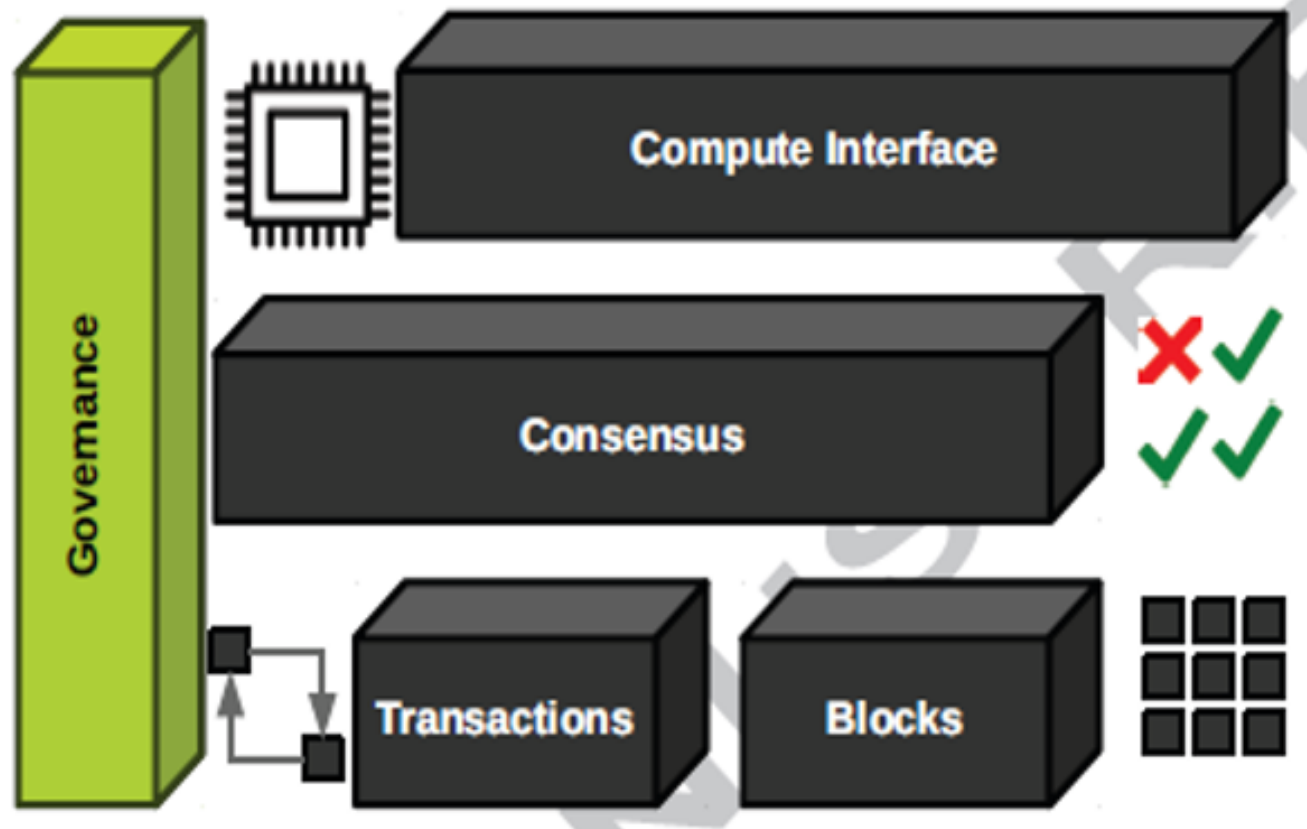

Figure 1

AN OVERVIEW OF BLOCKCHAIN ARCHITECTURE[4] 


\section{BLOCKCHAIN USE CASE SELECTION FRAMEWORK}

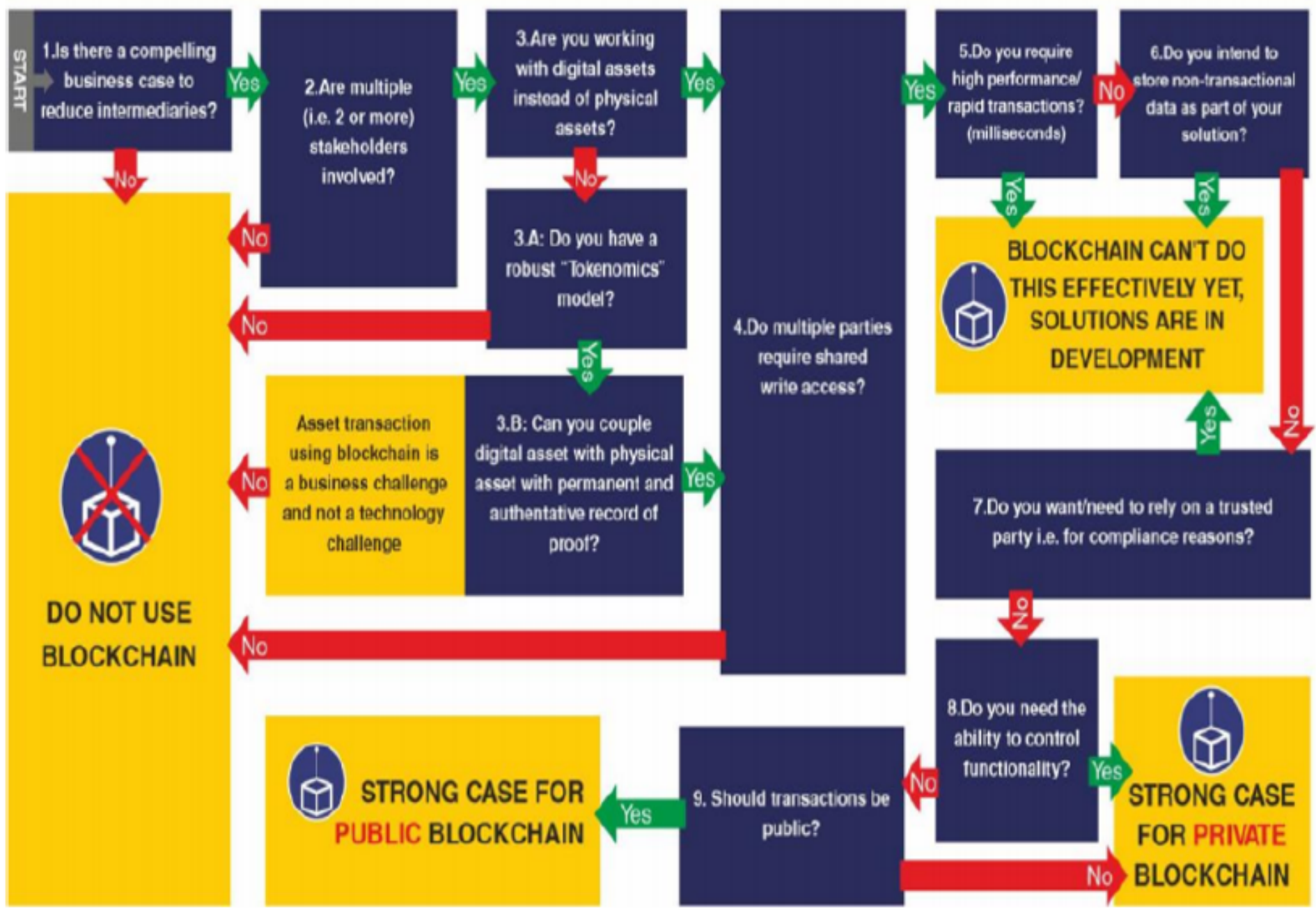

Figure 2

FRAMEWORK FOR BLOCKCHAIN USE CASE EVALUATION [5] 


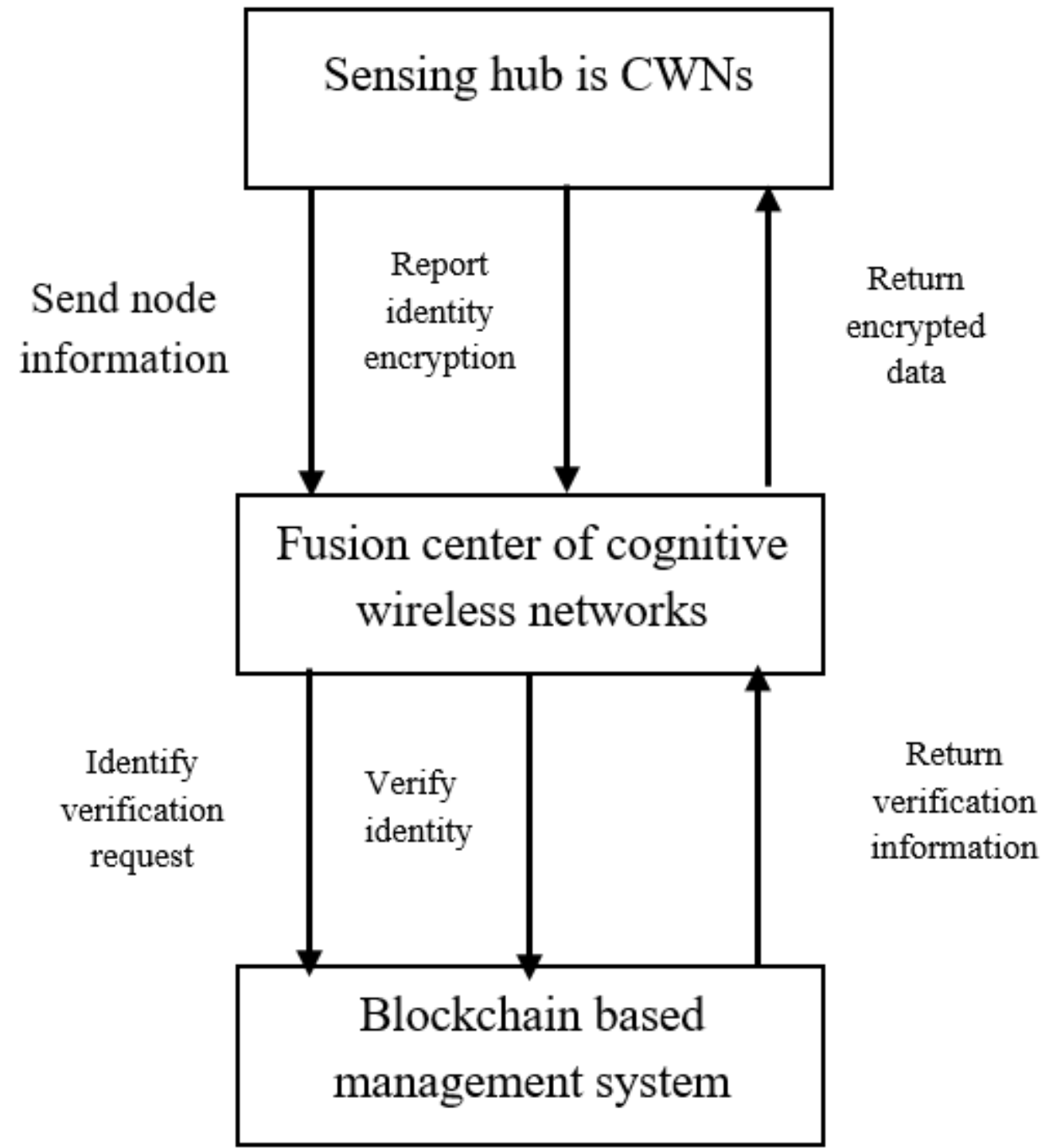

Figure 3

Security calculation construction of intellectual remote organization 


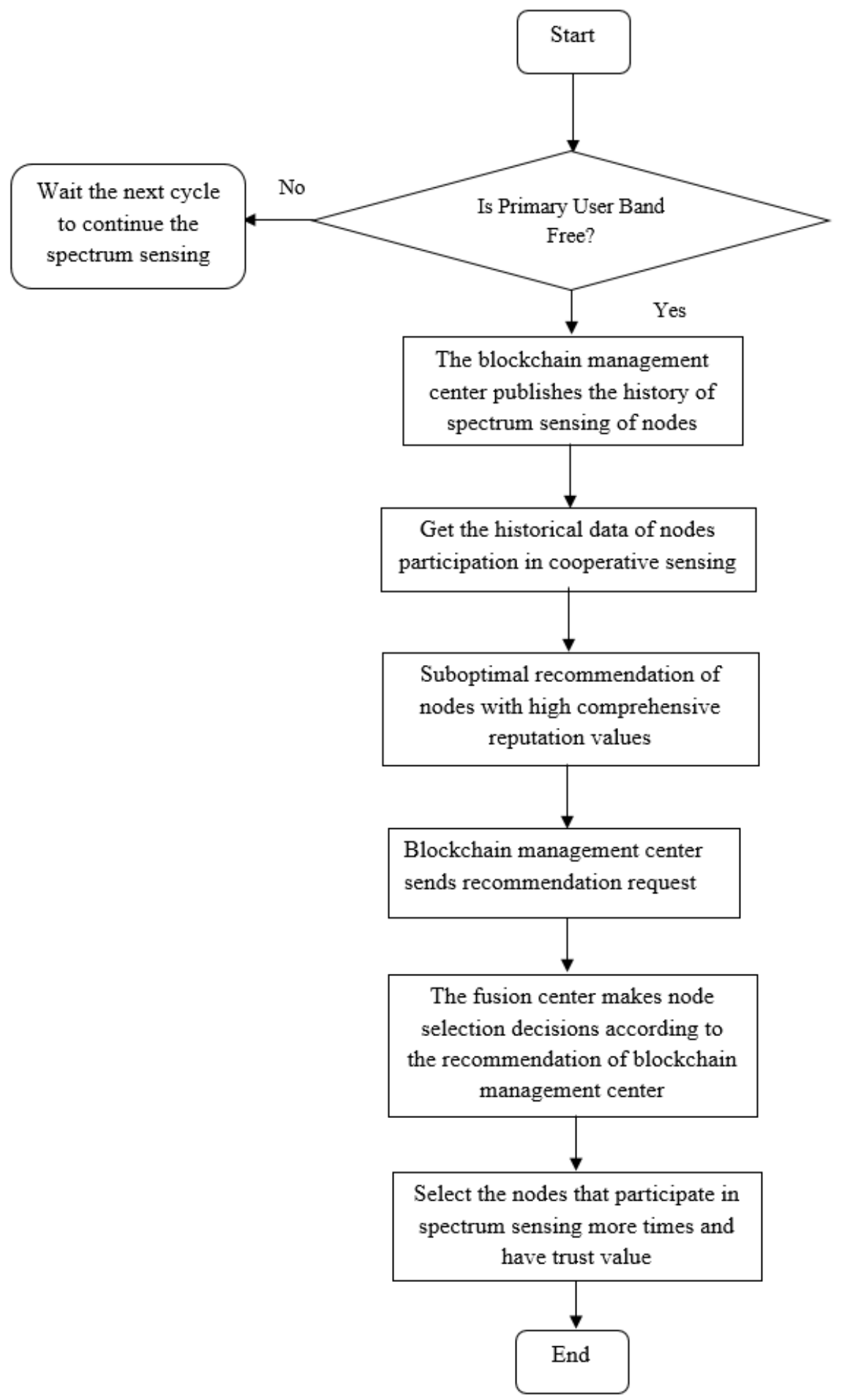

Figure 4

Secure Spectrum Sensing Algorithm Based on Blockchain In Cognitive Wireless Networks 


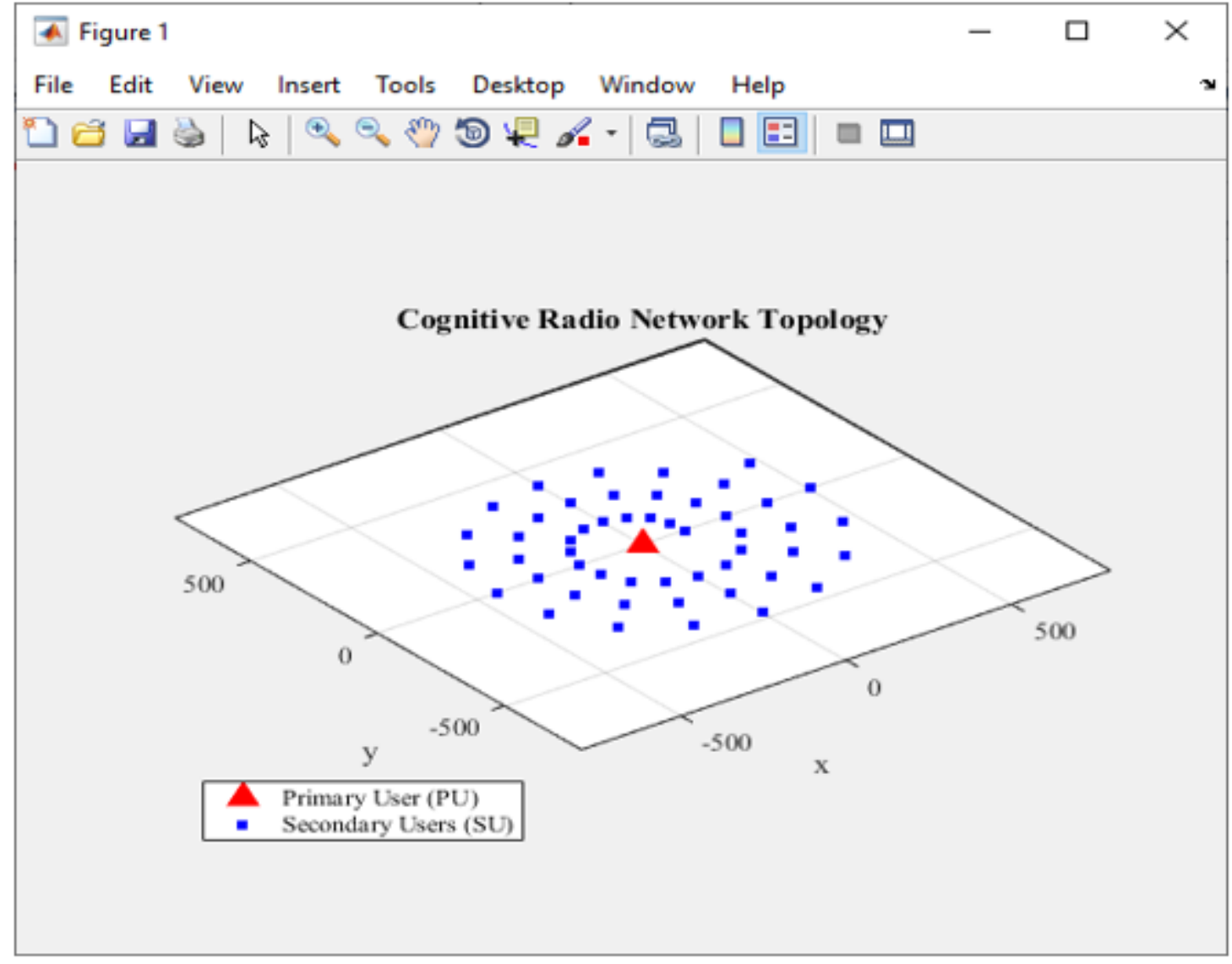

Figure 5

Cognitive Radio Topology

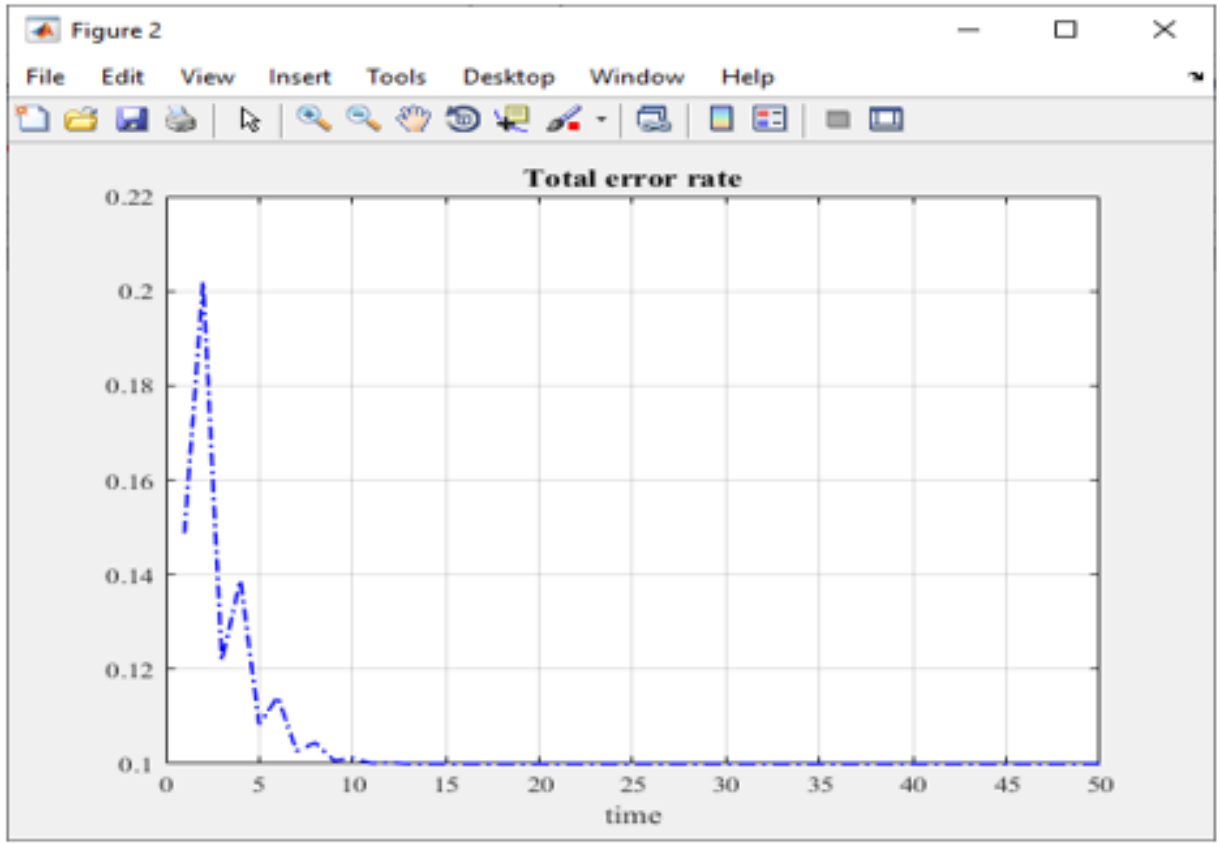

Figure 6

Total Error Rate 


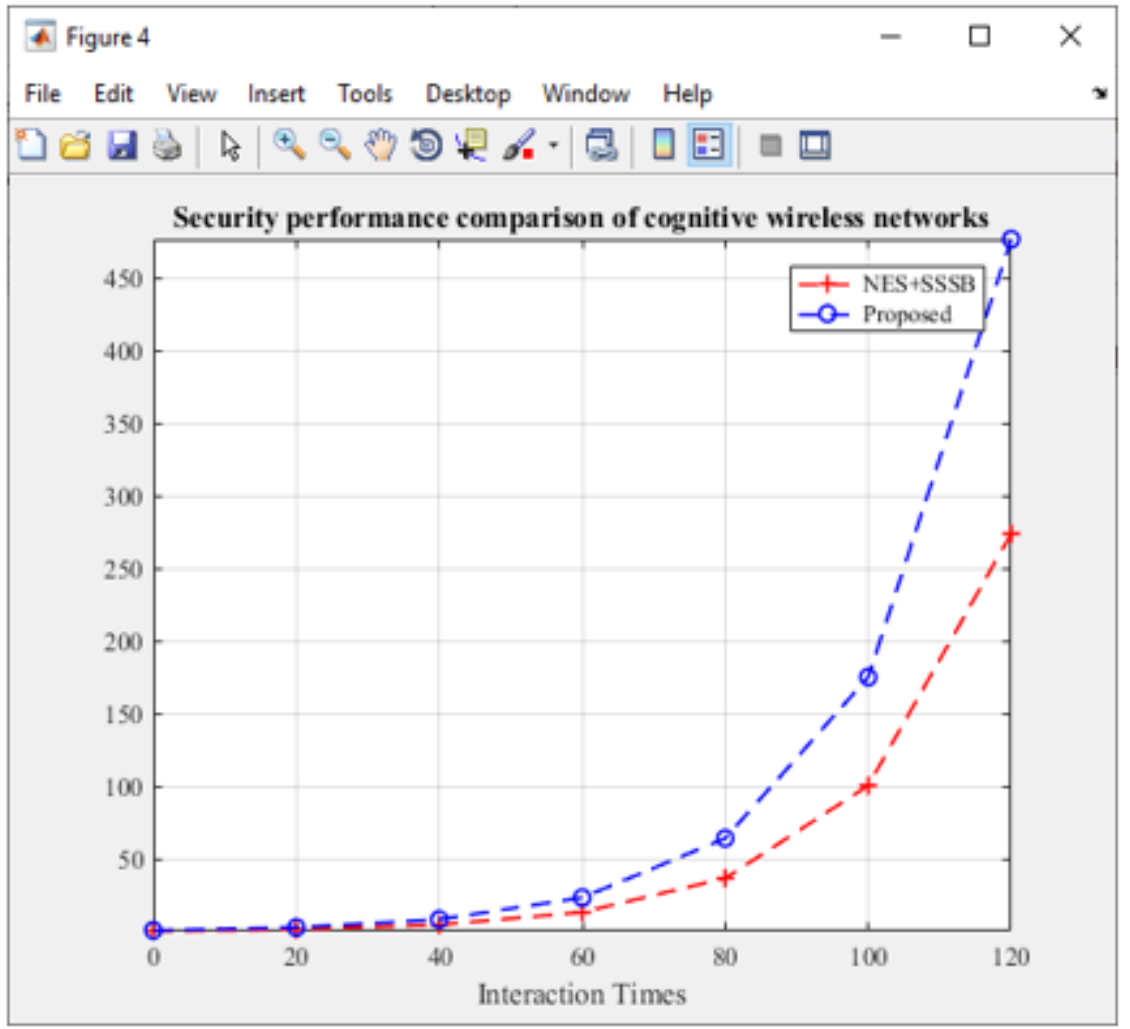

Figure 7

Comparative Analysis of Security in Cognitive Radio

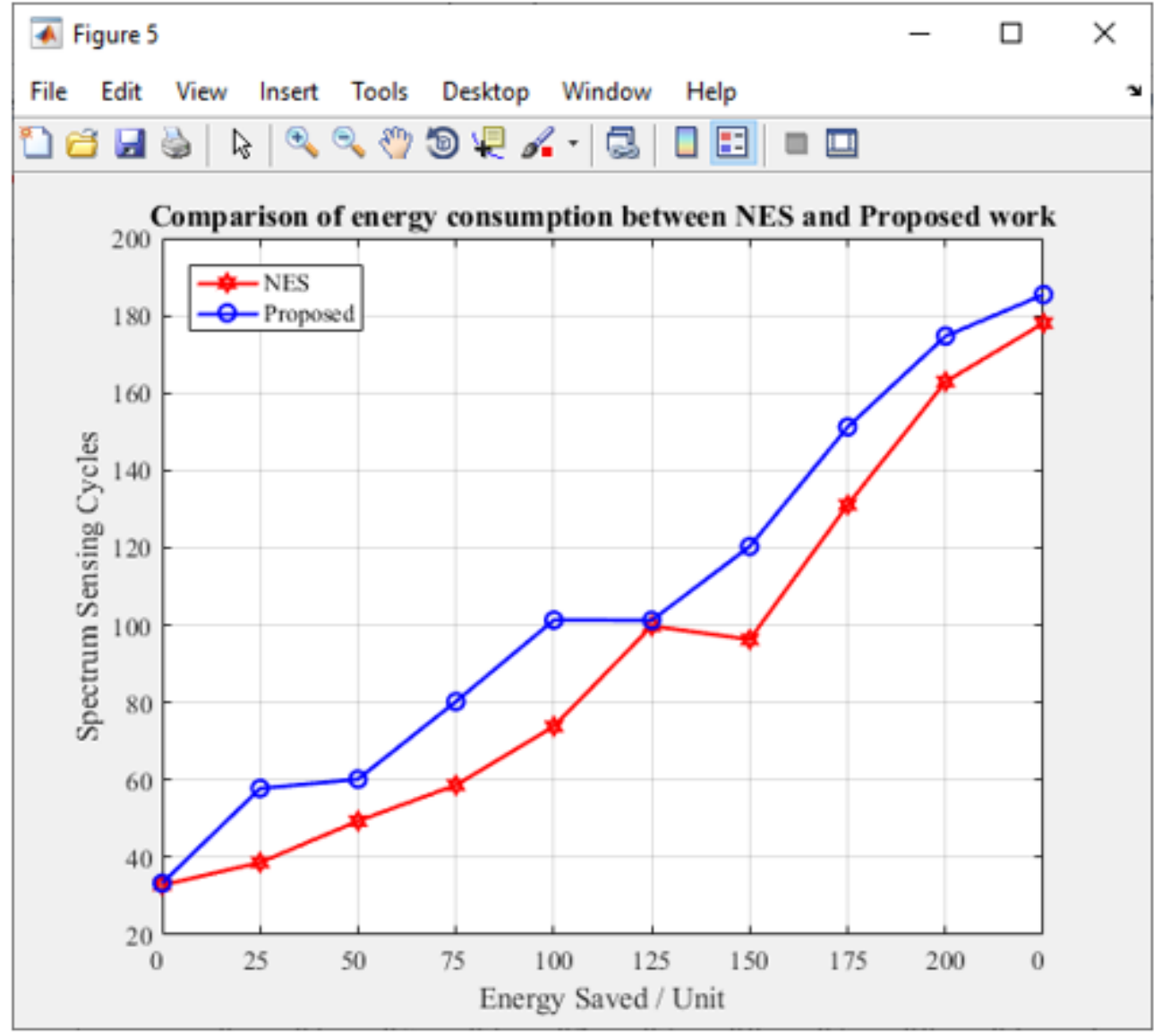


Figure 8

Comparative Analysis of Energy Consumption between Proposed and NES Algorithm 